\title{
ARTICLE Involvement of dopamine, but not norepinephrine, in the sex- specific regulation of juvenile socially rewarding behavior by vasopressin
}

\author{
Remco Bredewold (iD) ${ }^{1}$, Nara F. Nascimento ${ }^{2}$, Grace S. Ro ${ }^{2}$, Shannon E. Cieslewski ${ }^{2}$, Christina J. Reppucci (iD) and Alexa H. Veenema ${ }^{1}$
}

Social play is a highly rewarding behavior displayed mostly during the juvenile period. We recently showed that vasopressin V1a receptor (V1aR) blockade in the lateral septum (LS) enhances social play in male juvenile rats, but reduces it in females. Here, we determined whether the LS-AVP system modulates dopamine (DA) and/or norepinephrine (NE) neurotransmission in the LS to regulate social play behavior in sex-specific ways. Using microdialysis combined with retrodialysis, we demonstrated that both LS-AVP administration and social play exposure increased extracellular LS-DA release in females, but not in males. Pharmacological blockade of LS-DA receptors reduced social play in both sexes, but required a higher dose in females. This suggests that baseline LS-DA release is sufficient for social play in males, while increased LS-DA release is necessary for social play in females.

Administration of a V1aR antagonist into the LS inhibited the social play-induced increase in extracellular LS-DA release in females. Furthermore, co-administration of the DA agonist apomorphine prevented the LS-V1aR blockade-induced decrease in social play in females. This suggests that LS-V1aR blockade reduces social play in females by dampening the rise in LS-DA release. Extracellular LS-NE release was enhanced in response to pharmacological manipulations of the LS-AVP system and to social play in males and/or females, but pharmacological blockade or stimulation of LS-NE receptors did not alter social play in either sex. Overall, we define a mechanism by which the LS-AVP system alters LS-DA neurotransmission differently in males than females resulting in the sex-specific regulation of juvenile social play behavior.

Neuropsychopharmacology (2018) 43:2109-2117; https://doi.org/10.1038/s41386-018-0100-2

\section{INTRODUCTION}

Social play is a highly motivating and rewarding behavior [1, 2] that is prominently displayed during the juvenile phase in most mammalian species [3, 4]. Children diagnosed with autism spectrum disorder (ASD) show impaired social play [5], which may be due to disrupted reward-seeking tendencies $[6,7]$. Furthermore, ASD shows strong sex differences in incidence, symptoms severity, and treatment responses [8]. Overall, this indicates that research into the neurobiological underpinnings of the naturally rewarding aspects of social play and its regulation in both sexes is essential and may eventually inform therapeutic strategies.

Research on juvenile male rats suggests the involvement of dopamine (DA) and norepinephrine (NE) in the rewarding aspects of social play behavior. For example, systemic administration of drugs enhancing DA signaling increased reinforcement responding for social play, and these effects were blocked by administration of non-specific DA receptor antagonists [9]. Moreover, systemic administration of the NE reuptake inhibitor atomoxetine decreased responding for social play and decreased the expression of social play [9]. Despite a few studies using local manipulation of the DA and NE systems [10,11], little is known about the sites of action of DA and NE signaling in the brain involved in social play behavior. In addition, all studies thus far focused on male rats and it is unclear whether DA and NE are similarly involved in social play behavior in females.

We recently showed that social play behavior is regulated by the lateral septum (LS) in both male and female juvenile rats [1214]. The LS receives dopaminergic input from the ventral tegmental area (VTA) and adrenergic input from the locus coeruleus $[15,16]$. Electrophysiological stimulation of the VTA excites LS neurons and application of DA receptor antagonists in the LS inhibits this effect [17]. However, bath application of DA or $\mathrm{NE}$ reduced the firing rate of a majority of spontaneously active LS neurons [18]. Therefore, it is likely known that the effects of DA and NE in the LS are dependent on additional inputs to the LS [19]. These inputs may include vasopressinergic efferents from the bed nucleus of the stria terminalis and medial amygdala [20-22]. In support, bath application of arginine vasopressin (AVP) reduced the DA-induced or NE-induced inhibition of spontaneous LS neuronal activity [18]. This suggests that LS-AVP modulates LS-DA and LS-NE signaling. Here, we hypothesized that this modulation occurs during social play behavior.

We previously demonstrated that the LS-AVP system regulates social play behavior in sex-specific ways. Specifically, pharmacological blockade of the AVP V1a receptor (V1aR) in the LS increased social play behavior in juvenile male rats but decreased it in females $[12,13]$. However, it is unclear what the underlying

\footnotetext{
${ }^{1}$ Department of Psychology, Neurobiology of Social Behavior Laboratory, Michigan State University, East Lansing, MI, USA and ${ }^{2}$ Department of Psychology, Neurobiology of Social Behavior Laboratory, Boston College, Chestnut Hill, MA, USA

Correspondence: Remco Bredewold (bredewol@msu.edu)
}

Received: 12 March 2018 Revised: 8 May 2018 Accepted: 16 May 2018

Published online: 22 May 2018 
mechanisms are. Using in vivo microdialysis combined with retrodialysis, we determined whether LS-AVP administration, LSV1aR blockade, and social play exposure are associated with changes in extracellular LS-DA and LS-NE release in male and female juvenile rats. On the basis of these changes, we then used a pharmacological approach to determine the causal involvement of LS-DA and LS-NE neurotransmission on social play behavior.

\section{MATERIALS AND METHODS}

Animals

Male and female Wistar rats (23-day-old) were obtained from Charles River (Raleigh, NC, USA) and maintained under standard laboratory conditions $(12 \mathrm{~h}$ light/dark cycle, lights off at 1400 hours, $22^{\circ} \mathrm{C}, 50 \%$ humidity, food and water ad libitum). Rats were housed in same-sex groups of four in standard rat cages $\left(48 \times 27 \times 20 \mathrm{~cm}^{3}\right)$ unless otherwise mentioned. The experiments were conducted in accordance with the guidelines of the $\mathrm{NIH}$ and approved by the Institutional Animal Care and Use Committee at Michigan State University and at Boston College.

Extracellular DA and NE release in the LS of juvenile male and female rats in response to LS-AVP system manipulations and social play exposure

The effect of LS-AVP administration (experiment 1), social play exposure (experiment 2), and local $\mathrm{V} 1 \mathrm{aR}$ antagonist administration combined with social play exposure (experiment 3 ) on extracellular DA and NE release in the LS of 32-day-old male and female rats was determined using intracerebral microdialysis with (experiment 1 and 3) or without (experiment 2) retrodialysis. All microdialysis experiments took place in the home cage of individually housed experimental rats. At 28 days of age, experimental rats were housed individually in a standard rat cage with bedding that was not changed for the remainder of the experiments. Experimental rats were familiarized with the microdialysis setup 1 day after being housed individually. At 30 days of age, experimental rats were anesthetized with isoflurane (Butler Schein Animal Health, Dublin, OH, USA) and mounted on a stereotaxic frame (Kopf Instruments, Tujunga, CA, USA) with the tooth bar set at $-4.5 \mathrm{~mm}$. The u-shaped microdialysis probes (Brainlink, Groningen, The Netherlands) were unilaterally implanted into the LS according to Lukas et al. [23] at coordinates $0.4 \mathrm{~mm}$ caudal to bregma, $-1.5 \mathrm{~mm}$ lateral to the midline, $5.6 \mathrm{~mm}$ beneath the surface of the skull at an angle of $10^{\circ}$ to avoid damage to the sagittal sinus. The probes were filled with sterile Lactated Ringer's solution ( $\mathrm{pH} 7.4$ ) and fixed to the skull with three stainless steel screws and dental cement. Two 5-cm-long pieces of polyethylene tubing (PE 20, Plastics One, Roanoke, VA, USA) filled with Ringer's solution were connected to the inflow and the outflow of the probe and fixed with dental cement. After 1 day of surgery, probes were flushed with Ringer's and rats were familiarized with the microdialysis procedure. After 2 days of surgery, microdialysis probes were perfused with Ringer's $(3.0 \mu \mathrm{l} /$ min, $\mathrm{pH}$ 7.4) using a microinfusion pump. After a stabilizing period of $2 \mathrm{~h}, 6$ consecutive 10-min dialysates were collected. For experiment $1-3$, dialysates 1 and 2 were taken under baseline (undisturbed) conditions. For experiment 1, dialysate 3 was taken at the end of a 10-min retrodialysis period with synthetic AVP (Sigma; $1 \mu \mathrm{g} / \mathrm{ml}$; this concentration will result in $\sim 2 \mathrm{ng}$ of AVP delivered locally into the LS during a 10-min retrodialysis period, based on Engelmann et al. (1992)), and dialysates 4, 5, and 6 were taken thereafter. For experiment 2, dialysate 3 was taken at the end of the 10-min social play test, and dialysates 4, 5, and 6 were taken thereafter. For the social play test, each experimental rat was exposed to an age-matched and sex-matched socially housed unfamiliar rat for a 10-min period. For experiment 3, dialysate 3 was taken at the end of a 10-min retrodialysis period with the V1aR antagonist $\mathrm{d}\left(\mathrm{CH}_{2}\right)_{5}-\left(\mathrm{Tyr}(\mathrm{Me})^{2}\right) \mathrm{AVP}$ [24] $(10 \mu \mathrm{g} / \mathrm{ml}$; this concentration will result in $\sim 20 \mathrm{ng}$ of $\mathrm{V} 1 \mathrm{aR}$ antagonist delivered locally into the LS during a 10-min retrodialysis period, based on Engelmann et al. (1992)), dialysate 4 was taken at the end of a second $10-\mathrm{min}$ retrodialysis period with the $\mathrm{V} 1 \mathrm{aR}$ antagonist combined with 10-min social play exposure, and dialysates 5 and 6 were taken thereafter. Microdialysates were immediately frozen on dry ice and stored at $-45^{\circ} \mathrm{C}$. DA and NE concentrations were quantified in $10 \mu \mathrm{l}$ dialysates by HPLC with tandem mass spectrometry (MS/MS) detection, using deuterated internal standards of the analytes (BrainsOnline, San Francisco), as described previously [25]. Rats were killed with $\mathrm{CO}_{2}$ and proper probe placement in the LS (Fig. 1a) was checked on Nissl-stained $30 \mu \mathrm{m}$ cryocut coronal brain sections. Rats with incorrect probe placement were removed from the analysis. The final number per group is indicated in the legends of Figs. 2 and 3. During microdialysis, the percentage of social play duration of male and female rats was $8.34 \pm 1.53$ and $7.27 \pm 1.64$ respectively, which is similar to our previous microdialysis study in juvenile rats [14].

Effects of pharmacological manipulations of DA or NE neurotransmission in the LS on social play behavior in juvenile male and female rats

To determine the causal involvement of extracellular LS-DA and LS-NE release patterns on social play behavior, various drugs that alter DA or NE neurotransmission were administered in the LS. After 5 days of daily handling to familiarize them to the injection procedure, experimental rats ( 30 days of age) were anesthetized with isoflurane (Butler Schein Animal Health, Dublin, $\mathrm{OH}$, USA) and mounted on a stereotaxic frame with the tooth bar set at -4.5 $\mathrm{mm}$. Guide cannulae (22 gauge; Plastics One, Roanoke, VA, USA) were implanted $2 \mathrm{~mm}$ dorsal to the medial part of the LS according to Veenema et al. [12] at coordinates $0.4 \mathrm{~mm}$ caudal to bregma, $-1.0 \mathrm{~mm}$ lateral to the midline and $3.6 \mathrm{~mm}$ ventral to the surface of the skull at an angle of $10^{\circ}$ from the midsagittal plane to avoid damage to the sagittal sinus. Cannulae were fixed to the skull with three stainless steel screws and dental cement and closed with a dummy cannula (Plastics One, Roanoke, VA, USA). After surgery, rats were housed individually. After 2 days, rats received a $0.5 \mu \mathrm{l}$ injection of either Ringer's solution (vehicle), the non-specific DA receptor antagonist cis-(z)-flupenthixol dihydrochloride (Sigma-Aldrich F114; 15 or $30 \mu \mathrm{g} / 0.5 \mu \mathrm{l}$ ), the $\mathrm{V} 1 \mathrm{aR}$ antagonist $\left(\mathrm{dCH}_{2}\right)_{5}-\left[\operatorname{Tyr}\left(\mathrm{Me}^{2}\right)\right]$ AVP $(10 \mathrm{ng} / 0.5 \mu \mathrm{l})$, a combination of the V1aR antagonist and the DA agonist ( $r)-(-)$-apomorphine hydrochloride (Tocris $2074 ; 0.2$ or $2.0 \mu \mathrm{g} / 0.5 \mu \mathrm{l}$ ), the norepinephrine agonist DL-norepinephrine hydrochloride (Sigma-Aldrich A7256; 10 or $30 \mathrm{nmol} / 0.5 \mu \mathrm{l}$ ), the alpha adrenergic receptor antagonist phentolamine hydrochloride (Sigma-Aldrich P7547; 1.0 or $5.0 \mu \mathrm{g} / 0.5 \mu \mathrm{l}$ ), or the beta adrenergic receptor antagonist (s)-(-)-propranolol hydrochloride (Sigma-Aldrich P8688; 2.0 or 5.0 $\mu \mathrm{g} / 0.5 \mu \mathrm{l}$ ). Rats were tested for social play 20 min later. Apomorphine hydrochloride and norepinephrine hydrochloride were dissolved in Ringer's solution containing $0.1 \%$ ascorbic acid. Drug doses were chosen based on previous microinjection studies showing behavioral effects [11, 26-30]. Behavioral testing occurred at the beginning of the dark phase (between 1400 hours and 1500 hours) and under red light conditions. Each experimental rat was exposed to an age-matched and sex-matched socially housed unfamiliar rat for a $10-\mathrm{min}$ period. Tests were videotaped for subsequent analysis of behavior by a researcher blinded to the treatment conditions using JWatcher (http://www. jwatcher.ucla.edu/). Social play duration of the experimental rats was scored according to Veenema and Neumann [31] and consisted of the time spent in playful social interactions including nape attacks, pinning and supine poses. The number of nape attacks, pinning and supine poses was also scored. After the experiments, rats were euthanized with $\mathrm{CO}_{2}$, and charcoal was injected as a marker to check proper cannula placement (Fig. 1b) on Nissl-stained $30 \mu \mathrm{m}$ cryocut coronal brain sections. Rats with 

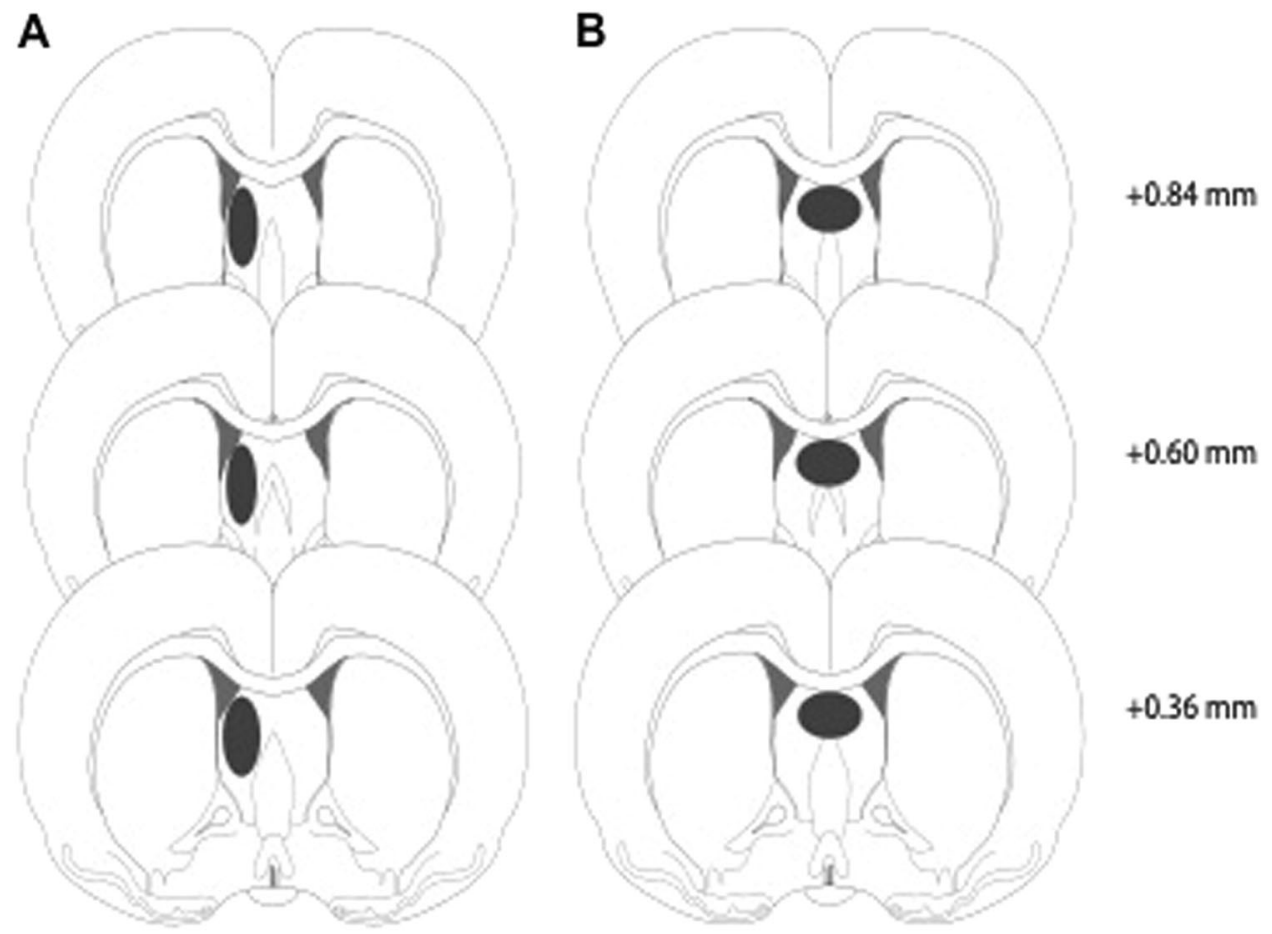

Fig. 1 Microdialysis probe and microinjection placements in the lateral septum of male and female juvenile rats. Schematic representation of coronal brain sections (from Paxinos and Watson, 2007) indicating in blue the areas marking correct placement of a microdialysis probe (a) or a microinjection (b) in the lateral septum. Microdialysis probe placement was aimed at the area of the LS showing high V1aR binding density in juvenile male and female rats [40]. Cannula placement was aimed at the medial LS in order to target both hemispheres of the LS. Distance from Bregma is indicated on the right

incorrect probe placement were removed from the analysis. The final number per group is indicated in the bars of Figs. 4 and 5.

\section{Statistics}

The percent of baseline DA release and NE release was analyzed using two-way ANOVAs for repeated measures (sex $\times$ dialysate). An LSD post hoc test was run when significant sex $\times$ dialysate interaction effects were found. When only a significant dialysate effect was found, a one-way ANOVA for repeated measures was run by sex to test for dialysate effects within each sex, followed by LSD post hoc testing. Absolute DA and NE concentrations were analyzed for sex effects using Student's $t$-tests. Drug treatment effects were analyzed using two-way ANOVAs (sex $\times$ drug) or oneway ANOVA (drug) followed by Bonferroni post hoc tests. Data are presented as mean+SEM. Significance was set at $p<0.05$.

\section{RESULTS}

Sex-specific effects of AVP and social play exposure on extracellular LS-DA release in juvenile rats

$L S$-DA release increased in females, but not in males, in response to $L S$ AVP treatment. The percent of baseline LS-DA release increased in response to LS-AVP administration in females but not in males (sex $\times$ dialysate effect: $F_{(5,75)}=5.60, p<0.001$ ). Specifically, in females, DA release during and immediately after AVP administration was significantly higher compared to baseline (LSD post hoc: $p$ $<0.005$, dialysate 3 vs. dialysates 1 and 2; $p<0.001$, dialysate 4 vs. dialysates 1 and 2; Fig. 2b). In contrast, in males, DA release did not change in response to AVP (LSD post hoc: $p>0.63$, dialysate 3 and 4 vs. dialysates 1 and 2; Fig. 2b). Hence, females showed a greater percent of baseline LS-DA release than males during and immediately after AVP administration (LSD post hoc: $p<0.01$, dialysate $3 ; p<0.005$, dialysate 4 ; Fig. $2 b$ ). There was no sex difference in absolute extracellular DA concentrations under baseline conditions ( $p=0.52$, average of dialysates 1 and 2 ) or during AVP administration ( $p=0.17, \mathrm{AVP}=$ dialysate 3 ; Fig. $2 \mathrm{c}$ ). However, absolute extracellular DA concentrations were significantly higher in females than males immediately after AVP administration ( $p<0.05$, post-AVP $=$ dialysate 4 ; Fig. $2 c)$.

$\angle S-D A$ release increased in females, but not in males, in response to social play exposure. The percent of baseline LS-DA release increased during social play exposure in females, but not in males (sex $\times$ dialysate effect: $F_{(5,65)}=3.19, p<0.05$ ). Specifically, in females, DA release during and immediately after social play exposure was significantly higher compared to all other dialysates (LSD post hoc: $p<0.05$, dialysates 3 and 4 vs. dialysates 1, 2, 5, and 6; Fig. 2e). In contrast, in males, DA release during social play exposure was not significantly different from any other dialysate (LSD post hoc: $p>$ 0.35 dialysate 3 vs. dialysate 1, 2, 4, 5, and 6; Fig. 2e). No sex difference was found for absolute extracellular DA concentrations under baseline conditions ( $p=0.19$, average of dialysates 1 and 2) or during social play exposure ( $p=0.89$, Play = dialysate 3 ; Fig. $2 f)$.

$L S-D A$ release did not change in response to $L S-V 1 a R$ antagonist treatment. Although a main effect of dialysate was found for the percent of baseline LS-DA release (dialysate effect: $F_{(5,55)}=2.80, p$ $<0.05)$, subsequent testing per sex did not reveal a significant dialysate effect (dialysate effect: $F_{(5,30)}=1.51, p=0.22$ in females; $F_{(5,25)}=1.63, p=0.19$ in males; Fig. $\left.2 f\right)$. No sex difference was found for absolute DA concentrations under baseline conditions $(p=0.11$, average of dialysates 1 and 2 ) or during $\mathrm{V} 1 \mathrm{aR}$ antagonist treatment $(p=0.35, \mathrm{~V} 1 \mathrm{aRa}=$ dialysate $3 ; p=0.16, \mathrm{~V} 1 \mathrm{aRa}+\mathrm{Play}=$ dialysate 4; Fig. 2i).

Extracellular LS-NE release increased in male and female juvenile rats in response to LS-AVP treatment, social play exposure, and LS-V1aR antagonist treatment $L S-N E$ release increased in both sexes in response to LS-AVP treatment. The percent of baseline LS-NE release increased in 

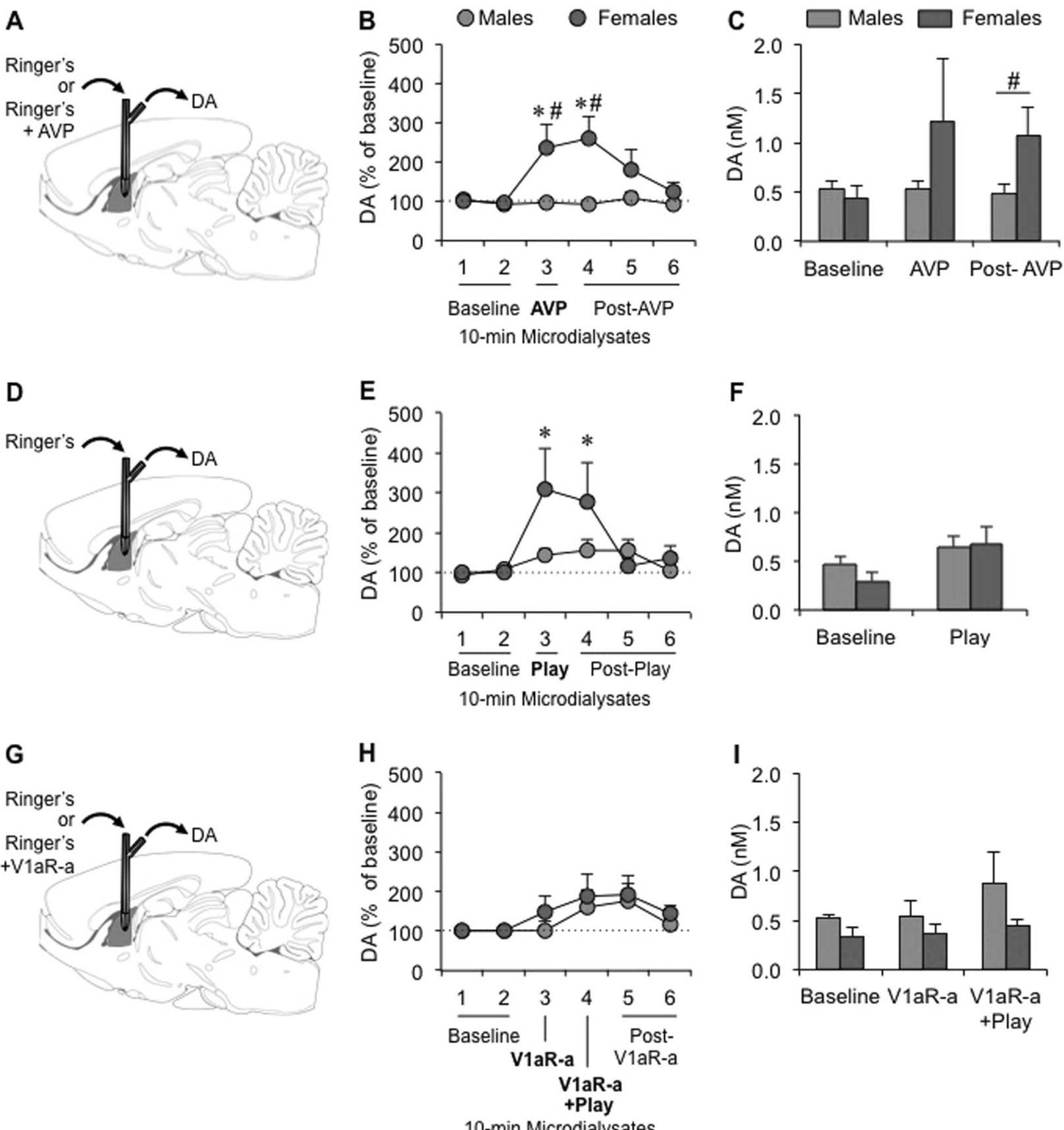

Fig. 2 Sex-specific effects of vasopressin (AVP) and social play exposure on extracellular dopamine (DA) release in the lateral septum (LS) of juvenile rats. DA release was measured in the LS under baseline conditions and in response to LS-AVP retrodialysis (males $n=11$, females $n=$ 6 ; a-c), social play exposure (males $n=9$, females $n=7 ; \mathbf{d}-\mathbf{f}$ ), and LS-V1aR antagonist (V1aR-a) retrodialysis (males $n=6$, females $n=7 ; \mathbf{g}-\mathbf{i})$. The percent of baseline DA release increased in females, but not in males, in response to AVP retrodialysis (b) and social play exposure (e), but did not change in response to $\mathrm{V} 1 \mathrm{aR}$ antagonist retrodialysis (h). Females showed higher DA concentrations than males after AVP administration (Post-AVP = dialysate 4; c) while no sex differences were observed under baseline conditions (Baseline = mean of dialysates 1 and $2 ; \mathbf{C}, \mathbf{f}, \mathbf{i})$, during social play exposure (Play=dialysate 3$)(\mathbf{f})$, or during V1aR antagonist retrodialysis (i). Data are presented as mean + SEM; Two-way ANOVA for repeated measures (sex $\times$ dialysate) followed by LSD post-hoc testing for $\mathbf{b}, \mathbf{e}$, and $\mathbf{h}$; Student's $t$-test for $\mathbf{c}, \mathbf{f}$, i; ${ }^{*} p<0.05$ vs. baseline (dialysates 1 and 2 ) in females, $\# p<0.05$ females vs. males

response to LS-AVP administration (dialysate effect: $F_{(5,90)}=5.00$; $p<0.001)$. Further statistical analysis by sex confirmed this effect in males (dialysate effect: $\left.F_{(5,45)}=3.41 ; p<0.05\right)$ with increased percent of baseline LS-NE release in response to AVP $(p<0.05$, dialysate 4 vs. dialysates 1 and 2; Fig. 3b), and approached significance in females (dialysate effect: $F_{(5,45)}=2.41 ; p=0.051$ ). No sex difference was found for absolute extracellular NE concentrations under baseline conditions or in response to AVP administration (Fig. 3c).

LS-NE release increased in both sexes in response to social play exposure. The percent of baseline LS-NE release increased in response to social play exposure (dialysate effect: $F_{(5,70)}=4.92 ; p<$ $0.005)$. Further statistical analysis by sex confirmed this effect in 

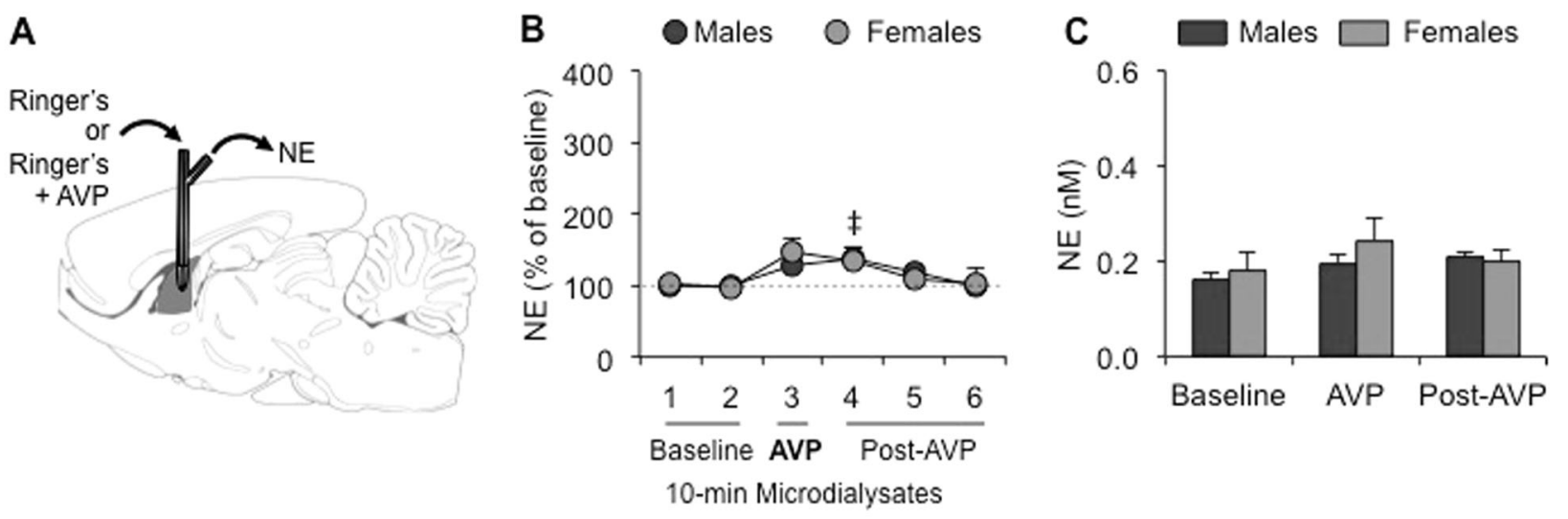

D

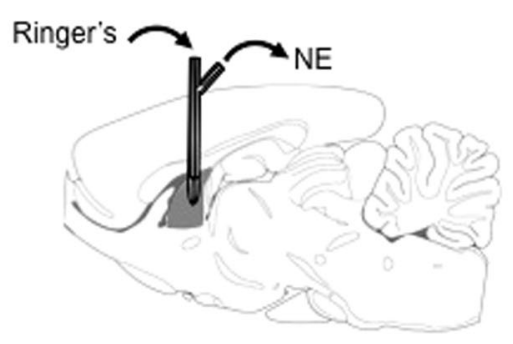

E
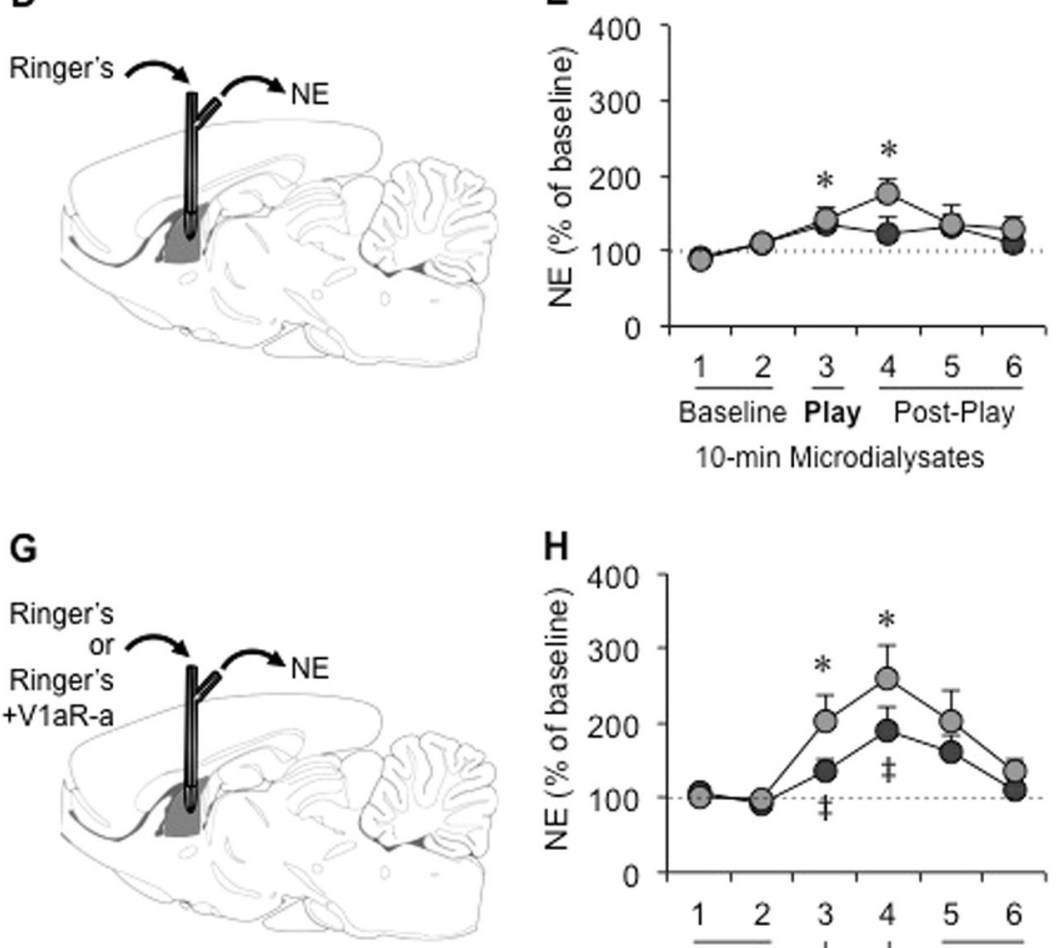

H

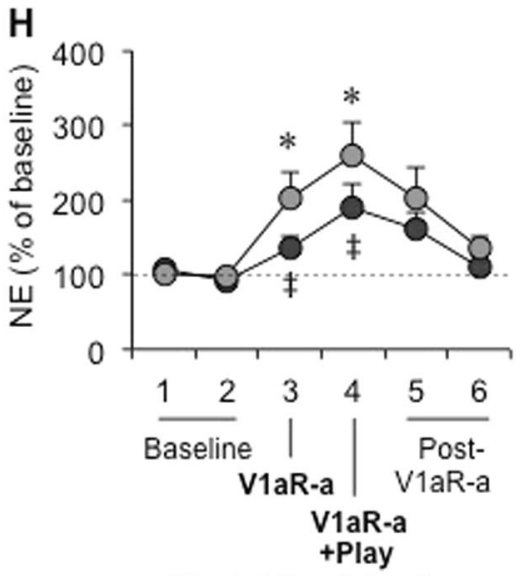

10-min Microdialysates
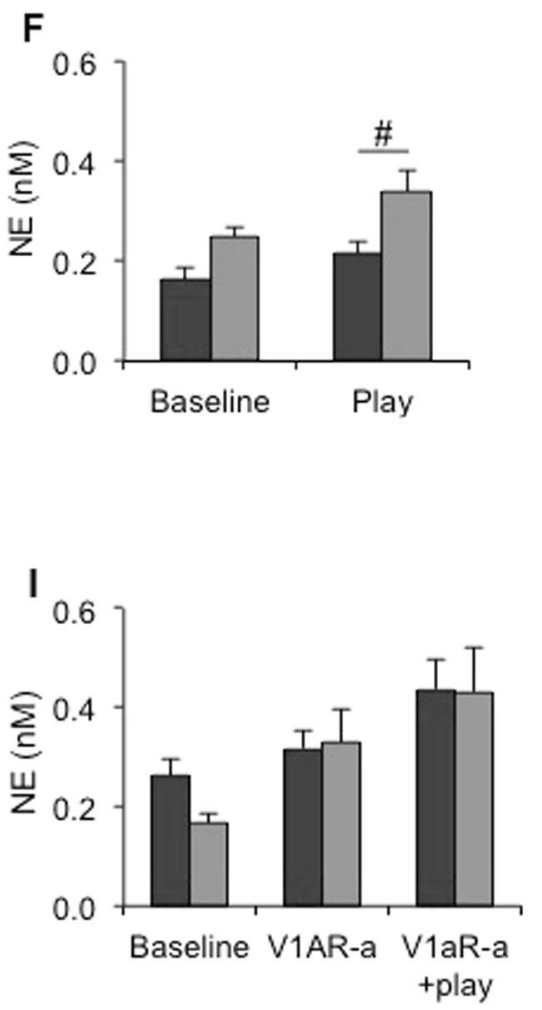

Fig. 3 Extracellular norepinephrine (NE) release in the lateral septum (LS) of juvenile male and female rats increased in response to vasopressin (AVP), social play exposure and V1aR antagonist (V1aR-a) treatment. NE release was measured in the LS under baseline conditions and in response to LS-AVP retrodialysis (males $n=10$, females $n=10 ; \mathbf{a}-\mathbf{c}$ ), social play exposure (males $n=8$, females $n=8$; $\mathbf{d}-\mathbf{f}$ ), and LS-V1aRa retrodialysis (males $n=9$, females $n=8 ; \mathbf{g}-\mathbf{i}$ ). The percent of baseline NE release increased in males and females in response to AVP retrodialysis (b), during social play exposure (e), and during V1aR antagonist retrodialysis (h). No sex differences in absolute NE concentrations were observed under baseline conditions (Baseline = mean of dialysates 1 and $2 ; \mathbf{c}$, $\mathbf{f}$, $\mathbf{i}$ ), but females showed higher absolute NE concentrations than males during social play exposure (Play = dialysate 3; f). Data are presented as mean+SEM; Two-way ANOVA for repeated measures (sex $\times$ dialysate) followed by one-way ANOVA for repeated measures by sex (dialysate) followed by LSD post-hoc testing for $\mathbf{b}$, $\mathbf{e}$, and $\mathbf{h}$; Student's $t$-test for $\mathbf{c}$, f, and $\mathbf{i} ; \neq p<0.05$, treatment effect in males, dialysate 3 vs. dialysate 2 (h) and dialysate 4 vs. dialysates 1 and 2 (b, h); ${ }^{*} p<0.05$, treatment effect in females, dialysate 3 vs. dialysate 1 (e), dialysate 3 vs. dialysate 1 and 2 (h), dialysate 4 vs. dialysates 1 and 2 (e, h); $\# p<0.05$ females vs. males (f)

males (dialysate effect: $\left.F_{(5,35)}=2.50 ; p<0.05\right)$ and in females (dialysate effect: $\left.F_{(5,35)}=3.26 ; p<0.05\right)$. Subsequent post hoc testing revealed that the increase in percent of baseline NE release in response to social play exposure approached significance in males ( $p=0.057$, dialysate 3 vs. dialysate $1 ; p=0.061$, dialysate 3 vs. dialysate 2 ; Fig. 3 e) and reached significance in females $(p<$ 0.05 , dialysate 3 vs. dialysates $1 ; p<0.05$, dialysate 4 vs. dialysates 1 and 2; Fig. 3e). No sex difference was found for extracellular NE 

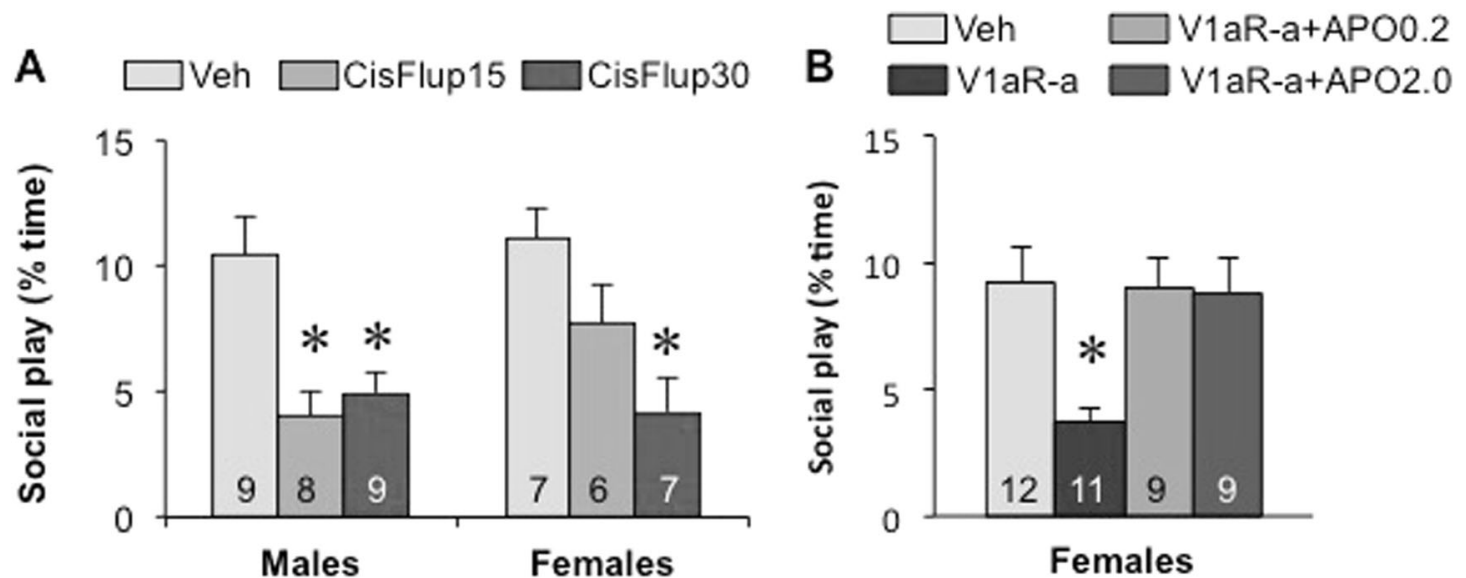

Females

Fig. 4 Involvement of the dopamine (DA) system in the lateral septum in the regulation of social play behavior in male and female juvenile rats. a Administration of the non-specific DA receptor antagonist cis-(z)-flupenthixol at either $15 \mu \mathrm{g} / 0.5 \mu \mathrm{l}$ (CisFlup15) or $30 \mu \mathrm{g} / 0.5 \mu \mathrm{l}$ (CisFlup30) reduced social play duration compared to vehicle treatment (Veh) in males, while only the higher dose (CisFlup30) reduced social play duration in females. $\mathbf{b}$ Administration of the V1aR antagonist $\left(\mathrm{d}\left(\mathrm{CH}_{2}\right)_{5}-\left[\mathrm{Tyr}(\mathrm{Me})^{2}\right] \mathrm{AVP}\right.$ at $10 \mathrm{ng} / 0.5 \mu \mathrm{l}$ (V1aR-a) reduced social play duration compared to vehicle treatment (Veh) in females, an effect that is prevented by co-administration of the DA agonist apomorphine at $2 \mu \mathrm{g} / 0.5 \mu \mathrm{l}$ (APO2) but not at $0.2 \mu \mathrm{g} / 0.5 \mu \mathrm{l}$ (APO0.2). Data are presented as mean + SEM; Two-way ANOVA (sex $\times$ treatment; a) or one-way ANOVA (treatment; b) followed by Bonferroni post-hoc testing. $\mathbf{a}{ }^{*} p<0.05$ vs. vehicle; $\boldsymbol{b}{ }^{*} p<0.05$ vs. vehicle, V1aR-a + APO0.2, and V1aR-a + APO2.0

concentrations under baseline conditions. However, absolute NE concentrations were higher in females than in males during social play $(p<0.05$; Fig. 3f).

$L S-N E$ release increased in both sexes in response to $L S-V 1 a R$ antagonist treatment. The percent of baseline NE release in the LS increased during V1aR antagonist treatment (dialysate effect: $\left.F_{(5,75)}=11.5 ; p<0.001\right)$. Further statistical analysis by sex confirmed this effect in males (dialysate effect: $F_{(5,40)}=5.23 ; p<0.001$ ) and in females (dialysate effect: $F_{(5,35)}=6.31 ; p<0.001$ ) with increased percent of baseline LS-NE release in response to the $\mathrm{V} 1 \mathrm{aR}$ antagonist (males: $p<0.05$, dialysate 3 vs. dialysate 2; females: $p<0.05$, dialysate 3 vs. dialysates 1 and 2; Fig. 3h) and in response to the $\mathrm{V} 1 \mathrm{aR}$ antagonist combined with social play exposure (both sexes: $p<0.05$, dialysate 4 vs. dialysates 1 and 2; Fig. $3 \mathrm{~h})$. No sex difference was found for absolute NE concentrations under baseline conditions or during V1aR antagonist treatment (Fig. 3i).

LS-DA signaling facilitates social play behavior in both sexes and plays a role in the LS-V1aR antagonist-induced decrease in social play behavior in females

Rats were administered the non-specific DA receptor antagonist cis-(z)-flupenthixol into the LS to determine the causal involvement of LS-DA release in the regulation of social play. LS-DA receptor blockade decreased social play duration (Treatment effect: $\left.F_{(2,40)}=13.5 ; p<0.001\right)$ at both doses in males $(p<0.01,15$ and $30 \mu \mathrm{g} / 0.5 \mu \mathrm{l}$ vs. vehicle), while it decreased social play duration only at the higher dose in females $(p<0.005,30 \mu \mathrm{g} / 0.5$ $\mu \mathrm{l}$ vs. vehicle; Fig. $4 \mathrm{a}$ ). The same pattern was seen for the number of nape attacks (Treatment effect: $F_{(2,40)}=13.6 ; p<0.001$ ) with LSDA receptor blockade decreasing the number of nape attacks at both doses in males ( $p<0.005,15$ and $30 \mu \mathrm{g} / 0.5 \mu \mathrm{l}$ vs. vehicle), while it decreased the number of nape attacks only at the higher dose in females $(p<0.005,30 \mu \mathrm{g} / 0.5 \mu \mathrm{l}$ vs. vehicle; Table S1). The number of pins was also affected by LS-DA receptor blockade (Treatment effect: $\left.F_{(2,40)}=6.80 ; p<0.005\right)$, resulting in fewer pins at the higher dose in females only $(p<0.05,30 \mu \mathrm{g} / 0.5 \mu \mathrm{l}$ vs. vehicle; Table S1).

To determine whether an increase in LS-DA signaling could prevent the decrease in social play behavior as previously seen in females after LS-V1aR blockade [12, 13], females were administered the V1aR antagonist into the LS in conjunction with the DA agonist apomorphine. Administration of the V1aR antagonist into the LS decreased social play duration in females (Treatment effect: $F_{(3,37)}=5.13 ; p<0.01$; Post hoc: $p<0.01$, V1aR-a vs. vehicle; $p<0.05$, V1aR-a vs. V1aR-a + AP00.2 and V1aR-a + APO2; Fig. 4b), while the combined administration of the V1aR antagonist with either dose of apomorphine did not alter social play duration in females (Post hoc: $p=1.00$, vehicle vs. V1aR-a + APO0.2 and V1aR-a + APO2; Fig. 4b). A similar pattern was seen for the number of nape attacks (Treatment effect: $F_{(2,37)}=5.18 ; p$ $<0.005)$ and the number of pins (Treatment effect: $F_{(2,37)}=4.33 ; p$ $<0.05)$, with fewer nape attacks and fewer pins on V1aR antagonist-treated females compared with females receiving the combined treatment of $\mathrm{V} 1 \mathrm{aR}$ antagonist and the high dose of apomorphine (nape attacks: $p<0.005$; pins: $p<0.05$ ) and compared with vehicle-treated females (pins: $p<0.05$; Table S2).

LS-NE signaling does not regulate social play behavior in either sex Administration of the non-specific NE agonist DL-norepinephrine hydrochloride, the alpha adrenergic receptor antagonist phentolamine, or the beta adrenergic receptor antagonist propranolol into the LS did not significantly alter social play duration (Fig. 5) nor the number of nape attacks, pins, and supine poses (Table S3, S4, S5) in males or in females. However, propranolol treatment induced a sex effect for the number of nape attacks (Sex effect: $\left.F_{(2,38)}=12.6 ; p<0.005\right)$, with fewer nape attacks in females than in males at both doses $(p<0.05$; Table S5).

\section{DISCUSSION}

This is the first in vivo study demonstrating that extracellular DA and NE release in the LS is modulated by the LS-AVP system and by social play exposure. Importantly, extracellular LS-DA release patterns were sex-specific as reflected by increased LS-DA release in response to LS-AVP administration and in response to social play exposure in females only. Notably, LS-V1aR blockade prevented the social play-induced increase in LS-DA release in females. Furthermore, pharmacological manipulations show that LS-DA neurotransmission facilitates social play behavior in both sexes and that enhancing LS-DA neurotransmission counteracts the inhibiting effects of LS-V1aR blockade on social play behavior in females. Finally, LS-AVP manipulations and social play exposure 
A

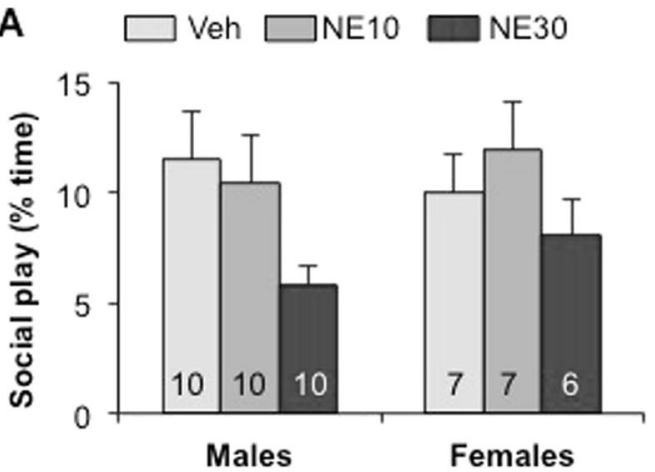

B $\square$ Veh $\square$ Phentolamine1 $\square$ Phentolamine5

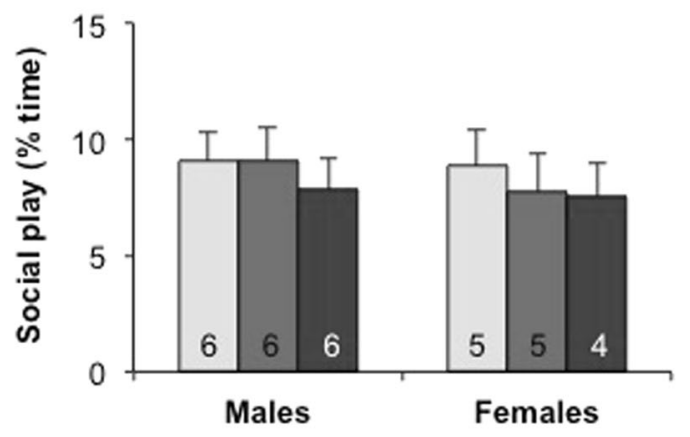

C $\square$ Veh $\square$ Propranolol2 $\square$ Propranolol5

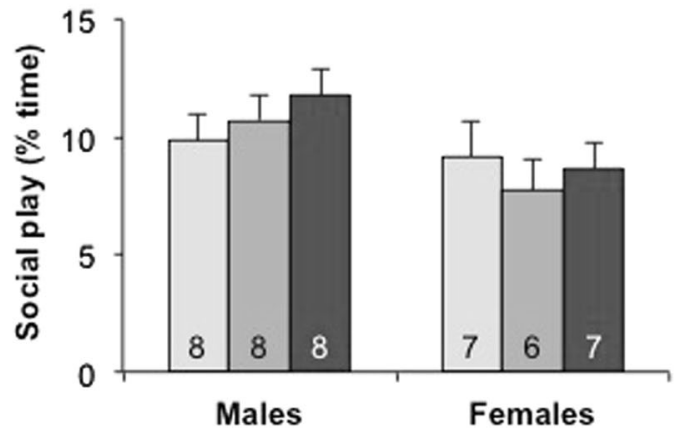

Fig. 5 No effects of manipulations of the norepinephrine (NE) system in the lateral septum on social play behavior in male and female juvenile rats. a Administration of NE at either the lower dose (10 nmol/0.5 $\mu \mathrm{l}$ Ringer's; NE10) or higher dose $(30 \mathrm{nmol} / 0.5 \mu \mathrm{l}$ Ringer's; NE30) did not significantly alter social play duration compared to vehicle treatment (Veh) in males and females. b Administration of the alpha adrenergic receptor antagonist phentolalamine at either the lower dose $(1 \mu \mathrm{g} / 0.5 \mu \mathrm{l}$ Ringer's) or higher dose $(5 \mu \mathrm{g} / 0.5 \mu \mathrm{l}$ Ringer's) did not significantly alter social play duration compared to vehicle treatment (Veh) in males and females. c Administration of the beta adrenergic receptor antagonist propranolol at either the lower dose $(2 \mu \mathrm{g} / 0.5 \mu \mathrm{l}$ Ringer's) or higher dose $(5 \mu \mathrm{g} / 0.5 \mu \mathrm{l}$ Ringer's) did not significantly alter social play duration compared to vehicle treatment (Veh) in males and females. Data are presented as mean+SEM; Two-way ANOVA (sex $\times$ treatment)

increased LS-NE release in males and/or females, but pharmacological manipulations of LS-NE neurotransmission did not alter social play duration in either sex. Altogether, these findings demonstrate a critical role for LS-DA, but not LS-NE, neurotransmission in the sex-specific regulation of social play behavior by the LS-AVP system.
Enhanced LS-DA release in response to LS-AVP administration or social play exposure in females

Although extracellular LS-DA release was similar between the sexes under baseline conditions, an increase in the percent of baseline LS-DA release in response to LS-AVP administration or social play exposure was seen in females only. Increased extracellular LS-DA release has been observed in response to systemic application of drugs of abuse such as cocaine in rats and morphine in mice [32, 33], suggesting a role for LS-DA neurotransmission in the positive and reinforcing effects of drug taking. This corresponds with other studies indicating that the LS is part of the brain reward system. For example, the LS is associated with electrical self-stimulation in rats and humans [34, 35] and is necessary for the formation of cocaine conditioned place preference in rats $[36,37]$. Importantly, the abovementioned studies used adult males and exposed them to nonsocial rewards. Our findings are the first to reveal a sex-specific pattern of LS-DA release during exposure to a social reward. This seems in line with a recent study showing increased Fos-activation of VTA-DA neurons in female, but not male, juvenile rats following social play exposure [38]. Together, these findings point towards distinct neural circuitries involved in social play and suggest a role for enhanced LS-DA neurotransmission in the rewarding aspects of social play behavior in females only.

LS-V1aR antagonist treatment prevents increase in LS-DA release in females

We further demonstrate that LS-V1aR blockade eliminates the sex difference in the percent of baseline in extracellular LS-DA release during social play exposure. Specifically, LS-V1aR antagonist administration did not induce a significant change in extracellular LS-DA release under baseline conditions in either sex and prevented the increase in LS-DA release seen in females exposed to social play. Moreover, enhancing LS-DA signaling by local administration of the DA agonist apomorphine prevented the decrease in social play behavior induced by LS-V1aR blockade in females. Together, this suggests that LS-V1aR antagonist reduces social play behavior in females by dampening the social playinduced rise in LS-DA release.

The mechanism by which the LS-AVP system modulates LS-DA release in females is presently unclear. Male and female rats show very dense $\mathrm{V} 1 \mathrm{aR}$ binding in the dorsal and intermediate $\mathrm{LS}$, with females having even denser binding than males [39, 40]. Some evidence in adult male mice suggests that a portion of $\mathrm{V} 1 \mathrm{aR}$ is located on terminals of dopaminergic neurons innervating the LS [41]. In detail, treatment with 6-hydroxydopamine, a neurotoxin that selectively destroys DA and NE neurons in the brain, reduced LS-DA and LS-NE concentrations as well as LS-AVP binding [41]. Importantly, treatment with 6-hydroxydopamine combined with the highly specific NE reuptake inhibitor desmethylimipramine, that prevents 6-hydroxydopamine to destroy NE neurons, reduced LS-DA concentrations as well as LS-AVP binding [41]. Thus, AVPinduced activation of LS-V1aR presynaptically located on dopaminergic terminals may be one plausible mechanism to explain the AVP-induced increase in LS-DA release as well as how LS-V1aR antagonist treatment could prevent the social play-induced increase in LS-DA release in juvenile female rats.

Lack of change in LS-DA release in response to LS-AVP administration or social play exposure in males

It may be surprising that no increase in LS-DA release was observed in males in response to LS-AVP administration or social play exposure. However, it is possible that fast and short increases in LS-DA release have been missed during the 10-min sampling time. Alternatively, baseline LS-DA release may be sufficient for social play in males. In support the non-specific DA receptor antagonist cis-(z)-flupenthixol decreased social play in males at a lower dose $(15 \mu \mathrm{g} / 0.5 \mu \mathrm{l})$ compared to females $(30 \mu \mathrm{g} / 0.5 \mu \mathrm{l})$. Thus, 
along with the sex-specific regulation of social play behavior by the LS-AVP and LS-glutamate systems [12-14], the observed sex difference in social play-induced LS-DA release, may be another indicator that social play behavior is regulated by distinct neural circuitries in males compared to females.

DA neurotransmission in the nucleus accumbens was also found to modulate social play behavior. Here, administration of a D1 receptor antagonist, a D2 receptor antagonist, or the non-specific DA receptor antagonist alpha-flupenthixol reduced social play behavior in juvenile male rats (females were not tested [11]). This indicates that DA acting at multiple brain sites is involved in the regulation of social play behavior. Interestingly, the nucleus accumbens and the LS share some of the same dopaminergic fibers originating from the VTA $[16,17]$. Thus, it is plausible that social play exposure triggers activation of VTA-DA neurons projecting to both the LS and nucleus accumbens. If so, we hypothesize that the social play-induced sex difference in extracellular DA release pattern as observed in the LS may also be present in the nucleus accumbens. Future studies may want to test this hypothesis that could provide further evidence of a sex-specific neural circuitry regulating the reinforcing properties of social play behavior.

No effect of LS-NE neurotransmission on social play behavior in either sex

Although extracellular LS-NE release increased following LS-AVP administration (males), LS-V1aR antagonist administration (both sexes), and social play exposure (females), pharmacological manipulations to enhance or reduce LS-NE signaling did not significantly alter social play duration in either sex. Despite the use of multiple drug doses, it cannot be excluded that additional drug doses or different types of drugs might alter social play duration. Indeed, enhancing NE signaling by using NE reuptake inhibitors reduced social play in juvenile male rats (females were not tested) when administered into other brain regions such as the basolateral amygdala and habenula [10]. Alternatively, the observed changes in extracellular LS-NE release may affect processes or behaviors that are relevant in the context of social play, a hypothesis that could be tested in future studies. Overall, the current findings suggest that NE neurotransmission in the LS does not play a major role in the regulation of social play behavior.

\section{CONCLUSIONS}

Here, we demonstrate that LS-DA, but not LS-NE, neurotransmission is involved in the sex-specific regulation of social play behavior by the LS-AVP system. These findings have broader significance. First, they provide further evidence of sex differences in the LS neural circuitry mediating a socially rewarding behavior. It would therefore be of interest to determine whether similar sex differences occur in the LS neural circuitry mediating other social and rewarding behaviors. Second, as discussed above, the sexspecific facilitation of extracellular DA release by social play exposure may not be limited to the LS because of VTA-DA collaterals projecting to both the LS and the nucleus accumbens $[16,17]$. Third, the sex-specific regulation of social play combined with the sex-specific facilitation of LS-DA release by LS-AVP provides new insights into the potential function of the sexually dimorphic extrahypothalamic AVP system, which is one of the most conserved sex differences in the brain of vertebrates [42, 43]. Last, elucidating the neural mechanisms mediating the sexspecific regulation of social reward in juveniles is an important step towards better understanding the neural basis of sex-biased social disorders such as ASD.

\section{ACKNOWLEDGEMENTS}

We thank Jennifer Schiavo and Michelle Verreij for technical assistance, Dr. Maurice Manning for providing the $\mathrm{V} 1 \mathrm{aR}$ antagonist, and members of the Veenema lab for input in previous versions of the manuscript. This research was supported by NSF IOS1253386 and NIH R01MH102456 to AHV.

\section{ADDITIONAL INFORMATION}

Supplementary Information accompanies this paper at (https://doi.org/10.1038/ s41386-018-0100-2).

Competing interests: The authors declare no competing interests.

Publisher's note: Springer Nature remains neutral with regard to jurisdictional claims in published maps and institutional affiliations.

\section{REFERENCES}

1. Trezza V, Baarendse PJ, Vanderschuren LJ. The pleasures of play: pharmacological insights into social reward mechanisms. Trends Pharmacol Sci. 2010;31:463-9.

2. Vanderschuren $L J$, Achterberg EJ, Trezza V. The neurobiology of social play and its rewarding value in rats. Neurosci Biobehav Rev. 2016;70:86-105.

3. Panksepp J. The ontogeny of play in rats. Dev Psychobiol. 1981;14:327-32.

4. Pellis SM, Pellis VC. Book The PlayfulBrain: Venturing to the Limits of Neuroscience. Oxford: Oneworld Publications; 2009.

5. Jordan R. Social play and autistic spectrum disorders: a perspective on theory, implications and educational approaches. Autism. 2003;7:347-60.

6. Kohls G, Chevallier C, Troiani V, Schultz RT. Social 'wanting' dysfunction in autism: neurobiological underpinnings and treatment implications. J Neurodev Disord. 2012;4:10.

7. Chevallier C, Kohls G, Troiani V, Brodkin ES, Schultz RT. The social motivation theory of autism. Trends Cogn Sci. 2012;16:231-9.

8. Rutter M, Caspi A, Moffitt TE. Using sex differences in psychopathology to study causal mechanisms: unifying issues and research strategies. J Child Psychol Psychiatry. 2003;44:1092-115.

9. Achterberg EJM, van Kerkhof LWM, Servadio M, van Swieten MM, Houwing DJ, Aalderink M, Driel NV, Trezza V, Vanderschuren LJMJ. Contrasting roles of dopamine and noradrenaline in the motivational properties of social play behavior in rats. Neuropsychopharmacology. 2016;41:858-68.

10. Achterberg EJM, Van Kerkhof LWM, Damsteegt R, Trezza V, Vanderschuren LJMJ. Methylphenidate and atomoxetine inhibit social play behavior through prefrontal and subcortical limbic mechanisms in rats. J Neurosci. 2015;35:161-169.

11. Manduca A, Servadio M, Damsteegt R, Campolongo P, Vanderschuren LJ, Trezza $V$. Dopaminergic neurotransmission in the nucleus accumbens modulates social play behavior in rats. Neuropsychopharmacology. 2016;41:2215-23.

12. Veenema AH, Bredewold R, De Vries GJ. Sex-specific modulation of juvenile social play by vasopressin. Psychoneuroendocrinology. 2013;38:2554-61.

13. Bredewold R, Smith CJ, Dumais KM, Veenema AH. Sex-specific modulation of juvenile social play behavior by vasopressin and oxytocin depends on social context. Front Behav Neurosci. 2014;16.8:216.

14. Bredewold R, Schiavo JK, van der Hart M, Verreij M, Veenema AH. Dynamic changes in extracellular release of GABA and glutamate in the lateral septum during social play behavior in juvenile rats: implications for sex-specific regulation of social play behavior. Neuroscience. 2015;307:117-27.

15. Lindvall $O$. Mesencephalic dopaminergic afferents to the lateral septal nucleus of the rat. Brain Res. 1975;87:89-95.

16. Lindvall O, Stenevi U. Dopamine and noradrenaline neurons projecting to the septal area in the rat. Cell Tiss Res. 1978;190:383-407.

17. Assaf SY, Miller JJ. Excitatory action of the mesolimbic dopamine system on septal neurones. Brain Res. 1977;129:353-60.

18. Joëls M, Urban IJ. Monoamine-induced responses in lateral septal neurons: influence of iontophoretically applied vasopressin. Brain Res. 1985:344:120-6.

19. Sheehan TP, Chambers RA, Russell DS. Regulation of affect by the lateral septum: implications for neuropsychiatry. Brain Res Brain Res Rev. 2004;46:71-117.

20. De Vries GJ, Buijs RM. The origin of the vasopressinergic and oxytocinergic innervation of the rat brain with special reference to the lateral septum. Brain Res. 1983;273:307-17.

21. De Vries GJ, Best W, Sluiter AA. The influence of androgens on the development of a sex difference in the vasopressinergic innervation of the rat lateral septum. Brain Res. 1983;284:377-80.

22. Caffé AR, van Leeuwen FW, Luiten PG. Vasopressin cells in the medial amygdala of the rat project to the lateral septum and ventral hippocampus. J Comp Neurol. 1987;261:237-52.

23. Lukas M, Bredewold R, Landgraf R, Neumann ID, Veenema AH. Early life stress impairs social recognition due to a blunted response of vasopressin release within the septum of adult male rats. Psychoneuroendocrinology. 2011;36:843-53. 
24. Manning M, Stoev S, Chini B, Durroux T, Mouillac B, Guillon G. Peptide and nonpeptide agonists and antagonists for the vasopressin and oxytocin V1a, V1b, V2 and OT receptors: research tools and potential therapeutic agents. Prog Brain Res. 2008;170:473-512.

25. Dwarkasing JT, Witkamp RF, Boekschoten MV, Ter Laak MC, Heins MS, van Norren $\mathrm{K}$. Increased hypothalamic serotonin turnover in inflammation-induced anorexia. BMC Neurosci. 2016;17:26.

26. Gulia KK, Kumar VM, Mallick HN. Role of the lateral septal noradrenergic system in the elaboration of male sexual behavior in rats. Pharmacol Biochem Behav. 2002;72:817-23.

27. Okada S, Yamaguchi N. Possible role of adrenoceptors in the hypothalamic paraventricular nucleus in corticotropin-releasing factor-induced sympathoadrenomedullary outflow in rats. Auton Neurosci. 2017;203:74-80.

28. Pal GK, Pal P, Raj SS, Mohan M. Modulation of feeding and drinking behaviour by catecholamines injected into nucleus caudatus in rats. Indian J Physiol Pharmacol. 2001;45:172-80.

29. Scotti MA, Lee G, Gammie SC. Maternal defense is modulated by beta adrenergic receptors in lateral septum in mice. Behav Neurosci. 2011;125:434-45.

30. Yilmaz MS, Myer BS, Feleder C, Millington WR. Blockade of \{alpha\}-adrenergic receptors in the preoptic area/anterior hypothalamus prevents lipopolysaccharide evoked hypotension. FASEB J. 2008;22:1227.8.

31. Veenema AH, Neumann ID. Maternal separation enhances offensive play-fighting, basal corticosterone and hypothalamic vasopressin mRNA expression in juvenile male rats. Psychoneuroendocrinology. 2009;34:463-7.

32. Reddy IA, Pino JA, Weikop P, Osses N, Sørensen G, Bering T, Valle C, Bluett RJ, Erreger K, Wortwein G, Reyes JG, Graham D, Stanwood GD, Hackett TA, Patel S, Fink-Jensen A, Torres GE, Galli A. Glucagon-like peptide 1 receptor activation regulates cocaine actions and dopamine homeostasis in the lateral septum by decreasing arachidonic acid levels. Transl Psychiatry. 2016;6:e809.

33. Sotomayor R, Forray Ml, Gysling K. Acute morphine administration increases extracellular DA levels in the rat lateral septum by decreasing the GABAergic inhibitory tone in the ventral tegmental area. J Neurosci Res. 2005;81:132-9.

34. Olds J, Milner P. Positive reinforcement produced by electrical stimulation of septal area and other regions of rat brain. J Comp Physiol Psychol. 1954;47:419-27.
35. Bishop MP, Elder ST, Heath RG. Intracranial self-stimulation in man. Science. 1963:140:394-6.

36. Luo AH, Tahsili-Fahadan P, Wise RA, Lupica CR, Aston-Jones G. Linking context with reward: a functional circuit from hippocampal $C A 3$ to ventral tegmental area. Science. 2011;333:353-7.

37. Sartor GC, Aston-Jones GS. A septal-hypothalamic pathway drives orexin neurons, which is necessary for conditioned cocaine preference. J Neurosci. 2012;32:4623-31.

38. Northcutt KV, Nguyen JM. Female juvenile play elicits Fos expression in dopaminergic neurons of the VTA. Behav Neurosci. 2014;128:178-86.

39. Smith CJ, Poehlmann ML, Li S, Ratnaseelan AM, Bredewold R, Veenema AH. Age and sex differences in oxytocin and vasopressin $\mathrm{V} 1 \mathrm{a}$ receptor binding densities in the rat brain: focus on the social decision-making network. Brain Struct Funct. 2017;222:981-1006.

40. Veenema AH, Bredewold R, De Vries GJ. Vasopressin regulates social recognition in juvenile and adult rats of both sexes, but in sex- and age-specific ways. Horm Behav. 2012;61:50-56.

41. Ishizawa H, Tabakoff B, Mefford IN, Hoffman PL. Reduction of arginine vasopressin binding sites in mouse lateral septum by treatment with 6hydroxydopamine. Brain Res. 1990;507:189-94.

42. DiBenedictis BT, Nussbaum ER, Cheung HK, Veenema AH. Quantitative mapping reveals age and sex differences in vasopressin, but not oxytocin, immunoreactivity in the rat social behavior neural network. J Comp Neurol. 2017;525:2549-70.

43. De Vries GJ, Panzica GC. Sexual differentiation of central vasopressin and vasotocin systems in vertebrates: different mechanisms, similar endpoints. Neuroscience. 2006;138:947-55.

44. Paxinos G, Watson C. The Rat Brain in Stereotaxic Coordinates. Academic Press, San Diego 2007

45. Engelmann M, Ludwig M, Landgraf R. Microdialysis administration of vasopressin and vasopressin antagonists into the septum during pole-jumping behavior in rats. Behav Neural Biol. 1992;58:51-7. 